\title{
Original
}

MOVILIDAD ESTUDIANTIL NACIONAL: INCLINACIÓN DE LOS UNIVERSITARIOS HACIA LA TOMA DE DECISIONES.

\section{MOVILIDAD ESTUDIANTIL NACIONAL: INCLINACIÓN DE LOS UNIVERSITARIOS HACIA LA TOMA DE DECISIONES}

\section{DOMESTIC STUDENT MOBILITY: STUDENTS' TENDENCY TOWARDS DECISION MAKING}

Itxayana Guadalupe Solís López*, Gladys Hernández Romero**

*Estudiante de la Licenciatura en Mercadotecnia. División Académica de Ciencias Económico Administrativas. Universidad Juárez Autónoma de Tabasco. Villahermosa, Tabasco, México. ORCID: https://orcid.org/0000-0002-3844-1499.

**Doctora en Educación. Profesora Investigadora. División Académica de Ciencias Económico Administrativas. Universidad Juárez Autónoma de Tabasco. Email: doctoragladys@hotmail.com. ORCID: https://orcid.org/0000-0002-9045-2937.

Dirección para recibir correspondencia: itxayanasolis@gmail.com

Fecha de recibido: 6 de mayo de 2019

Fecha de aceptación: 4 de junio de 2019

DOI: https://doi.org/10.19136/hitos.a25n72.3426

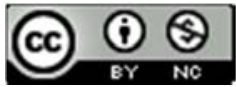

SOLÍS-LÓPEZ I. G., HERNÁNDEZ-ROMERO G. 
MOVILIDAD ESTUDIANTIL NACIONAL: INCLINACIÓN DE LOS UNIVERSITARIOS HACIA LA TOMA DE DECISIONES.

\section{RESUMEN}

La movilidad es un programa académico con el cual cuentan las Instituciones de Educación Superior (IES); éstos son revisados constantemente por evaluadores y acreditadores avalados, los cuales se encuentran en constante cambio, ocasionados por la globalización.

OBJETIVO: Exponer la percepción que tienen los estudiantes universitarios de los beneficios que se puedan obtener de la movilidad nacional e identificar el motivo por el cual elegirían la universidad a la cual postular.

MATERIAL Y MÉTODO: Se desarrolló una investigación cuantitativa, con un enfoque transversal, ya que solo se aplicará en un solo momento, no experimental, al igual que no probabilística, debido que se encuestaron a los sujetos a conveniencia.

RESULTADOS: Se muestra que el 81\% de los universitarios conoce la existencia del programa, pero que el $100 \%$ de ellos no ha vivido la experiencia, también sobresale que la información que tienen acerca de la movilidad es poca, debido a la falta de divulgación y conocimiento del programa con el que cuentan los universitarios.

CONCLUSIONES: Los estudiantes mantienen en consideración la oportunidad de hacer su pasantía en otras instituciones educativas, tales como: la Universidad Autónoma de Yucatán, Universidad de Guadalajara y la Universidad de Monterrey, lo que hace hincapié a su preferencia de permanecer dentro del país y no a viajar al extranjero.

PALABRAS CLAVE: Movilidad estudiantil. Enseñanza superior. Estudiante universitario. Enseñanza pública. 


\section{Original}

MOVILIDAD ESTUDIANTIL NACIONAL: INCLINACIÓN DE LOS UNIVERSITARIOS HACIA LA TOMA DE DECISIONES.

\section{ABSTRACT}

Mobility is an academic program in Higher Educations Institutions, which constantly is revised by evaluating and certifying organisms that are in continuous change due to globalization.

OBJECTIVE: To expose college students' perception on domestic mobility benefits and also to identify the reasons for selecting a host university.

MATERIAL AND METHOD: This is a quantitative, cross-sectional, non-probabilistic study with a convenience sample.

RESULTS: The results show that $81 \%$ of college students know the program pero $100 \%$ of them have not yet lived the experience. There is little information about the domestic mobility program due to the lack of promotion among the student population.

CONCLUSIONS: Students show interest about doing mobility in other Higher Education Institutions, such as: Universidad Autónoma de Yucatán, Universidad de Guadalajara and Universidad de Monterrey, highlighting the tendency towards domestic mobility instead of going abroad.

KEY WORDS: Student mobility. Higher education. College student. Public education.

\section{INTRODUCCIÓN}

Actualmente, se están viviendo cambios vertiginosos que antes no se tenían, muchos de ellos ocasionados por la globalización. Estos cambios impactan el día a día del ser humano, la educación superior no está exenta de éstos, y una prueba de ello son los programas académicos con los que cuentan las Instituciones de Educación Superior (IES) en apoyo a sus estudiantes, entre los que se están los diferentes tipos de beca que se ofrecen, Programa de Verano de Investigación Científica, Programa de Prácticas Profesionales y los Programas de Movilidad Académica. Éstos son revisados constantemente por evaluadores y acreditadores avalados por organismos tanto nacionales como internacionales, que buscan la mejora constante y permanente de los estudiantes (Universidad Juárez Autónoma de Tabasco, 2018, p. 16). 
MOVILIDAD ESTUDIANTIL NACIONAL: INCLINACIÓN DE LOS UNIVERSITARIOS HACIA LA TOMA DE DECISIONES.

Los programas de movilidad radican en el desplazamiento académico que realizan los estudiantes, profesores e investigadores a otros centros educativos, siendo principalmente de estudios superiores los que realizan con frecuencia este tipo de actividad, que es de carácter formativa. La movilidad académica, es una migración temporaria, la cual es el estar en un sitio por un periodo determinado, y al concluir, regresar a la región de origen.

Este proyecto consta de un periodo de durabilidad de seis meses, ya sea de carácter nacional, o internacional. Los alumnos deben cumplir con un cierto número de requisitos para poder ser acreedores a las becas de los Programas de Movilidad Estudiantil. En el caso de Universidad Juárez Autónoma de Tabasco (UJAT), actualmente cuenta sólo con el programa de movilidad académica nacional, al que pueden postularse los universitarios.

Los Sistemas Académicos Nacionales poseen como objetivo, que los alumnos obtengan conocimientos académicos, culturales, económicos y sociales; así como experiencias educativas trabajando con personas de otros Estados del país, con una visión diferente a lo que se está acostumbrado, y de esta forma adaptarse a los cambios sociales que ocurren constantemente.

La movilidad estudiantil no sólo se limita a otorgar experiencia académica, puesto que también "ofrece programas de estudio en áreas novedosas y necesarias para el crecimiento del país y con amplias perspectivas de ingreso al mercado de trabajo" (Fresan, 2009, p. 142), por medio del cual "ofrece nuevas dimensiones y oportunidades de cooperación científica de gran valor para los países en desarrollo" (Tejada, 2012, p. 3).

González (2011) considera que a la par de lo expresado anteriormente, las tecnologías de la información y de la comunicación han influido en el fomento de los flujos internacionales, en los que los estudiantes viajan a otro país o ciudad dentro de su mismo país y en algunos casos, se obligan a recibir en casa a estudiantes provenientes de esa misma universidad que visitaron, además permiten el intercambio de ideas y el almacenamiento de experiencias generando de esta manera una mayor interacción entre los estudiantes involucrados.

\section{La movilidad}

Cabe señalar, que la educación superior debe ser vista "como una de las principales riquezas que impulsa el desarrollo social, político y económico, por su contribución a la formación de SOLÍS-LÓPEZ I. G., HERNÁNDEZ-ROMERO G. 


\section{Original}

MOVILIDAD ESTUDIANTIL NACIONAL: INCLINACIÓN DE LOS UNIVERSITARIOS HACIA LA TOMA DE DECISIONES.

profesionales e investigadores, a la generación de nuevos saberes, la creatividad e innovación, al desarrollo científico y tecnológico del país" (Universidad Juárez Autónoma de Tabasco, 2015, p. 21).

El estar en constante movimiento, es una acción natural de los seres vivos, puesto que las personas diariamente se transportan a sus lugares de trabajos, casa, centros comerciales, y siempre regresan a un lugar, es decir su hogar.

El término movilidad, hace referencia a la actividad donde las personas migran de un lugar de origen en búsqueda del área en el cual desean residir temporal o definitivamente; de igual manera es utilizado para señalar la acción de desplazamiento realizado en entornos físico, ya sea de personas o mercancías.

\section{Movilidad académica}

Se refiere a cuando los alumnos, profesores e investigadores se trasladan a otras instituciones a cursar o impartir materias, dependiendo del rol que jueguen. La Asociación Nacional de Universidades e Instituciones de Educación Superior (ANUIES) señala en su página oficial que "en la movilidad estudiantil, los estudiantes de licenciatura y posgrado realizan prácticas, cursos cortos y residencias académicas fuera de su institución. Si la estancia se cumple en un país extranjero constituye un instrumento importante para la formación integral, profesional".

Dentro de las opciones con que cuentan los universitarios, se encuentra la que se desarrolla en el país de origen, es la denominada nacional. Cuando un universitario elige este tipo, es porque considera que le conviene por diversas razones no alejarse mucho de su lugar de residencia. Allende y Morones (2006, p. 2) señalan que, para lograr ser aceptado en estos programas, los aspirantes deben entre otros requisitos ser regulares; es decir, cursar las asignaturas señaladas en el tiempo marcado por la institución en la que están matriculados. Por lo tanto, el objetivo de este estudio es: Exponer la percepción que tienen los estudiantes universitarios de los beneficios que se puedan obtener de la movilidad nacional e identificar el motivo por el cual elegirían la universidad a la cual postular. 
MOVILIDAD ESTUDIANTIL NACIONAL: INCLINACIÓN DE LOS UNIVERSITARIOS HACIA LA TOMA DE DECISIONES.

\section{MATERIAL Y MÉTODO}

La UJAT es una de las principales Instituciones Públicas de Educación Superior, actualmente conformada por doce campus, ubicados a lo largo y ancho del territorio tabasqueño al SurSureste de los Estados Unidos Mexicanos. La matrícula estudiantil total es de 32,033 estudiantes distribuidos en los diferentes niveles académicos que oferta, como son: Técnicos, Licenciaturas, Especialidades, Maestrías y Doctorados (División Académica de Ciencias Económico Administrativas, 2018).

Tabla 1

Matrícula de estudiantes inscritos

\begin{tabular}{lc}
\hline Licenciado en Administración & 1658 \\
Licenciado en Contaduría Pública & 1474 \\
Licenciado en Economía & 268 \\
Licenciado en Relaciones Comerciales & 1 \\
Licenciado en Mercadotecnia & 896 \\
Licenciado en Administración (a distancia) & 122 \\
Licenciado en Contaduría Pública (a distancia) & 84 \\
Maestría en Administración y Dirección Estratégica & 17 \\
Maestría en Contaduría & 11 \\
Doctorado en Estudios Económicos-Administrativos & 18 \\
Maestría en Gerencia Pública y Gobierno & 14 \\
Maestría en Ciencias de Gestión del Desarrollo Regional & 3 \\
Maestría en Administración & 55 \\
Total & 4621
\end{tabular}

Fuente: 3er Informe de actividades División Académica de Ciencias económico Administrativo (2018).

La investigación se realizó en la División Académica de Ciencias Económico Administrativas, y el criterio para determinar la muestra de la elección de estudiantes de las licenciaturas en contaduría pública, administración, y mercadotecnia con edades promedio en el rango de 20 a 23, siendo elegidos los grupos de quinto semestre, (donde los alumnos cumplen con este requisito) sin importar género y dando por entendido que todos son alumnos regulares, ya que el programa exige un avance curricular mayor al 45\% para ser participante en dicha actividad.

Debido a que los requisitos solicitados por la universidad son:

- $\quad$ Tener promedio mínimo de 8.5.

- Haber cursado y aprobado el $45 \%$ de los créditos del plan de estudios.

- Ser alumno regular al momento de la postulación.

- Regresar a la UJAT a cursar el último ciclo escolar de la licenciatura, después de concluir la estancia.

- Postularse únicamente a dos universidades.

SOLÍS-LÓPEZ I. G., HERNÁNDEZ-ROMERO G. 


\section{Original}

MOVILIDAD ESTUDIANTIL NACIONAL: INCLINACIÓN DE LOS UNIVERSITARIOS HACIA LA TOMA DE DECISIONES.

Los estudiantes encuestados con un avance del 45\% deberían estar cursando el cuarto semestre de la licenciatura, es decir la mitad de la carrera, llegando apenas al porcentaje que pide la universidad para ser acreedor a participar en esta actividad, teniendo en cuenta que las licenciaturas tienen un periodo mínimo de duración de nueve semestres (cuatro años y medio).

La investigación es concluyente, con un enfoque cuantitativo, en el cual se requiere recolectar datos para cumplir con el objetivo planeado y comprobar las hipótesis, con un enfoque transversal, debido a que se ejecutará en un tiempo único de aproximadamente mes y medio; el periodo considerado fue del 18 de febrero al 12 de abril. Asimismo, se trata de una investigación no experimental, ya que no se manipuló ninguna variable, toda vez que fue tomada en cuenta la percepción de los encuestados; y finalmente no probabilística, porque se seleccionaron los sujetos a entrevistar a conveniencia.

El instrumento utilizado para la medición, consistió en un cuestionario semiestructurado de diez preguntas aplicado a los universitarios, quienes de buena manera aceptaron responder las preguntas planteadas.

Para el análisis de esta investigación, se utilizó una hoja de cálculo, donde se mostraron los datos de forma sistematizada, para su posterior teorización enmarcadas en conceptualizaciones respaldadas por especialistas en el tema.

\section{RESULTADOS}

Cuando se les cuestionó a los participantes si conocían el Programa Institucional, el 81\% respondió afirmativamente (ver figura 1).

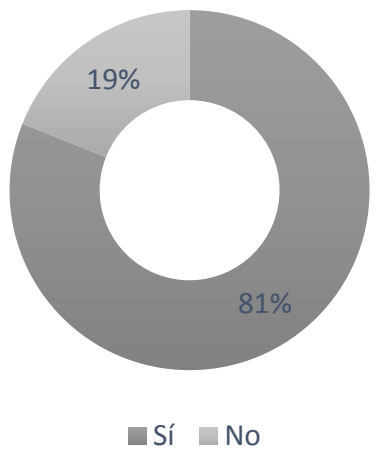

Figura 1. ¿Conoce el programa de movilidad nacional?

Fuente: Elaboración propia. 


\section{Original}

MOVILIDAD ESTUDIANTIL NACIONAL: INCLINACIÓN DE LOS UNIVERSITARIOS HACIA LA TOMA DE DECISIONES.

A la pregunta sobre si han participado en la movilidad, el 100\% de los alumnos respondió que no ha participado. De igual forma, cuando se les preguntó a los participantes si pensaban que esta actividad era necesaria para la formación educativa, el 90\% respondió afirmativamente, añadiendo que era un tipo de enseñanza diferente.

Los universitarios, señalan que les gustaría aplicar para las ciudades de Mérida, Guadalajara o Monterey (62\%), Ciudad de México (26\%), y el resto, 12\% desea ir a Puebla.

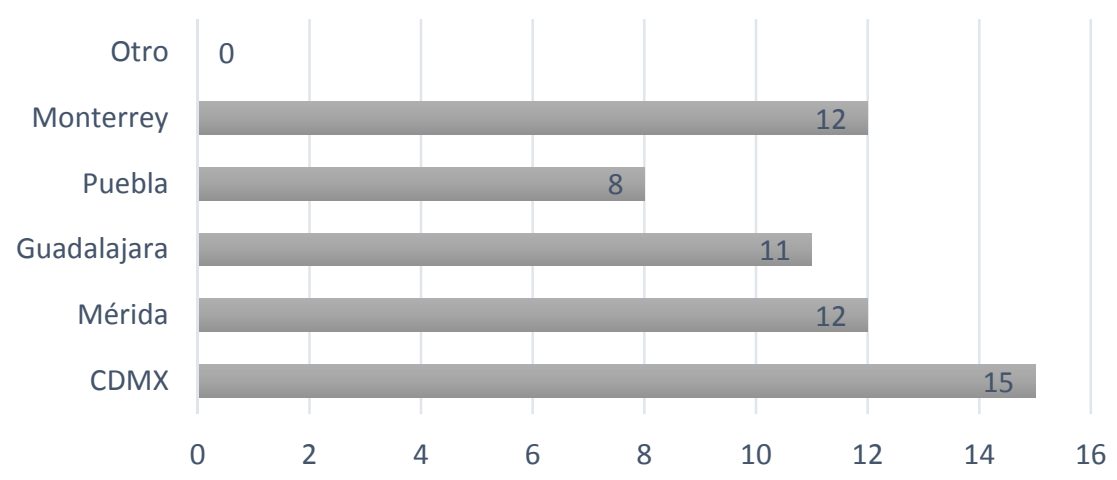

Figura 2. Estado al que le gustaría aplicar.

Fuente: Elaboración propia.

Estos resultados contrastan con lo respondido al cuestionamiento sobre en qué Institución de Educación Superior desean realizar su pasantía, el 34\% señala a la Universidad Nacional Autónoma de México (UNAM), a la que desea asistir sólo el $26 \%$ de los encuestados. La Universidad Autónoma de Yucatán (UADY), la Universidad de Guadalajara (UDG) y la Universidad de Monterrey (UDEM) fueron señaladas como opción para el 48\%, ubicándose en las ciudades que fueron elegidas por el $62 \%$ de los universitarios, $14 \%$ se decanta por la Benemérita Universidad Autónoma de Puebla (BUAP), la cual fue elegida por el 12\% de los participantes.

Al cuestionarles, a quien recurrirían a solicitar información acerca de esta actividad, el 71\% contestó que al personal administrativo, el 22\% dijo que a los docentes, y el $7 \%$ que recurriría a un compañero con mayor avance curricular. 
MOVILIDAD ESTUDIANTIL NACIONAL: INCLINACIÓN DE LOS UNIVERSITARIOS HACIA LA TOMA DE DECISIONES.

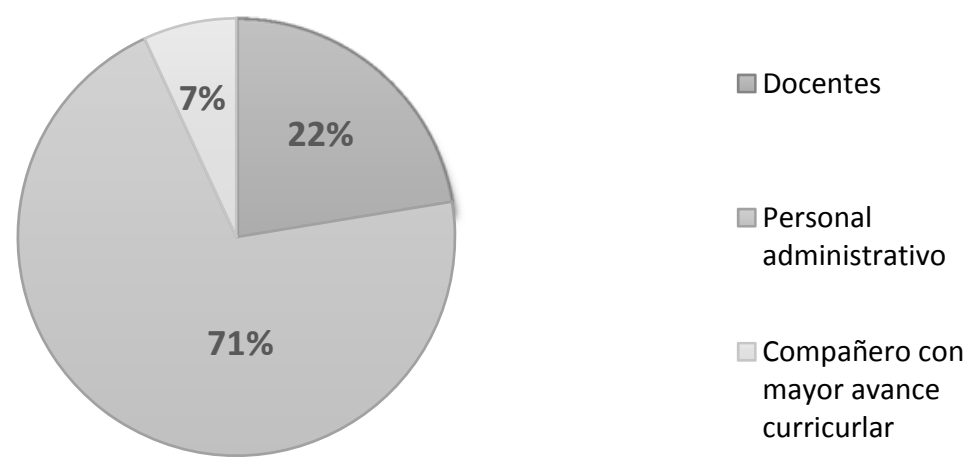

Figura 3. ¿A quién solicitaría información?

Fuente: Elaboración propia.

Los universitarios contestaron en su mayoría (83\%), que los beneficios que le traerían al participar en el programa son de carácter académico, porque existen tipos de enseñanza, calidad educativa, métodos de evaluación y aprendizaje, que podrían ser de provecho para ellos.

Al preguntar cuál sería el motivo por el que eligieron la universidad, el $41 \%$ de alumnos respondió que por el prestigio de la universidad, el 31\% por el lugar en que se encuentra, el 7\% por la familiaridad con la universidad, y el 21\% contestó que por la recomendación de profesores, amigos, o familiares.

\section{DISCUSIÓN}

Al inicio de la licenciatura (en el curso obligatorio de inducción) se les informa sobre los programas académicos con los que cuenta la universidad. Los alumnos encuestados que contestaron que no conocen el programa, puede ser debido a que no llegaron el día del curso, no les interesó acerca de los temas que se estaban tratando, o simplemente no prestaron atención.

Al concursar para ser parte de esta experiencia, es una oportunidad que tienen quienes reúnen los requisitos, mismos que son conocidos por todos los universitarios tabasqueños que asisten a esta institución, ya que estos requisitos se proporcionan en el curso de inducción, al igual que aparecen en la página web de la universidad.

SOLÍS-LÓPEZ I. G., HERNÁNDEZ-ROMERO G. 


\section{Original}

MOVILIDAD ESTUDIANTIL NACIONAL: INCLINACIÓN DE LOS UNIVERSITARIOS HACIA LA TOMA DE DECISIONES.

Belvis, Pineda y Moreno, consideran que con la movilidad se "pretende la armonización de las estructuras de la enseñanza superior y un sistema común de créditos que pretendan la promoción de la movilidad y la supresión de obstáculos para el ejercicio libre de la misma por los estudiantes" (2007, p. 1).

En el análisis de las opiniones, queda claro que muchos de ellos no conocen en que ciudad se localiza la universidad a la que pretenden asistir, lo que sí se pudo constatar es, que prefieren estar dentro del mismo país, que viajar a uno extranjero.

Los estudiantes son el motivo de ser de esta universidad (que a la par realiza actividades de investigación y de difusión), siendo parte importante del ser y quehacer universitario, se trabaja con un alto compromiso, buscando que vean en el país una oportunidad para desarrollarse académicamente, y que tengan una visión objetiva para proponer soluciones de frontera a los problemas nacionales.

Reflexionar sobre la formación de recursos humanos de alto nivel, desemboca frecuentemente en la siguiente cuestión: ¿Es mejor enviar a los jóvenes a estudiar al extranjero o educarlos en su propio país? Por una parte, se admite sin resistencia que estudiar en una universidad de primer nivel en los países más desarrollados es ventajoso. Por lo tanto, se cuestionan los diferenciales de costos, se denuncian los riesgos de Brain Drain y se critican los posibles efectos enajenadores de la educación en el exterior en la relación que el estudiante mantiene con su país de origen y su cultura (Schwartzman, 2009, p. 63).

La UJAT no cuenta con un Programa de Movilidad Internacional, este se tenía en años anteriores, actualmente se encuentra suspendido; la comunidad estudiantil espera que en los años siguientes vuelva a restablecer con normalidad.

La búsqueda información "Es un punto de partida para impulsar la movilidad de estudiantes y docentes como la homologación de la curricular, lo que permite generar igualdad de oportunidades para los estudiantes y egresados" (González y Salgado, 2016).

Al respecto, García de Fanelli señala que: "La movilidad de estudiantes constituye un fenómeno de gran complejidad porque encierra situaciones de muy distinta naturaleza y duración" (2009, p. 120). 


\section{Original}

MOVILIDAD ESTUDIANTIL NACIONAL: INCLINACIÓN DE LOS UNIVERSITARIOS HACIA LA TOMA DE DECISIONES.

\section{CONCLUSIONES}

Partiendo de la importancia que tiene para los universitarios participar en estos eventos, los resultados demuestran que el programa institucional es conocido por la mayoría de los alumnos, desde el primer día de clases se les brinda información de todos los programas académicos con los que cuenta la universidad; sin embargo, los alumnos que no están informados acerca de estas actividades, puede ser debido a que no asistieron los días en los que se brindó esta información, no prestaron la debida atención, o simplemente no les interesó el mensaje.

La movilidad es una acción necesaria para la formación educativa, al momento de considerar la actividad, se contemplan nuevas estructuras de enseñanza tanto académicas, culturales, sociales, experiencias educativas al poder trabajar con personas de otros estados, es por este motivo que muchos estudiantes desean aplicar para quedar seleccionados.

Los universitarios al examinar las instituciones donde desean realizar su pasantía, dentro de las más elegidas por la mayoría de los jóvenes se encuentran; la Universidad Autónoma de Yucatán, la Universidad de Guadalajara y la Universidad de Monterrey, el motivo de la elección es el prestigio y el lugar donde se encuentra.

Por otra parte, los participantes señalan que recurrirían a sus profesores para solicitar información, debido a que pueden proporcionar experiencias previas en las cuales recomiendan ciertas universidades, e investigadores en la cual realizar la pasantía.

Incluso, contando con información y el apoyo económico, los alumnos no buscan participar activamente en este programa, el cual, como se menciona anteriormente, logran proporcionar en múltiples beneficios que pueden contribuir en la futura vida laboral de los estudiantes.

\section{REFERENCIAS BIBLIOGRÁFICAS}

Asociación Nacional de Universidades e Instituciones de Educación Superior. (2018). Página oficial. Recuperado de http://www.anuies.mx/programas-y-proyectos/cooperacionacademica-nacional-e-internacional/cooperacion-acad.

Belvis, E., Pineda, P. y Moreno, M. (2007). La participación de los estudiantes universitarios en programas de movilidad: factores y motivos que la determinan. Revista Iberoamericana 


\section{Original}

MOVILIDAD ESTUDIANTIL NACIONAL: INCLINACIÓN DE LOS UNIVERSITARIOS HACIA LA TOMA DE DECISIONES.

de Educación 42(5). Recuperado de https://www.researchgate.net/publication/28156096_La_participacion_de_los_estudiante s_universitarios_en_programas_de_movilidad_factores_y_motivos_que_la_determinan.

De Allende, C. y Morones, G. (2006). Glosario de términos vinculados con la cooperación académica. México: Asociación Nacional de Universidades e Instituciones de Educación Superior.

Recuperado

de http://www.anuies.mx/media/docs/convocatorias/pdf/glosariocoopnal2-jul06.pdf.

Fresan Orozco, M. (2009). Impacto del Programa de Movilidad Académica en la formación integral de los alumnos. Revista de la Educación Superior, 38(151), 141-160. Recuperado de http://publicaciones.anuies.mx/pdfs/revista/Revista151_S4A1ES.pdf.

García de Fanelli, A. (2009). La movilidad Académica y estudiantil: reflexiones sobre el caso argentino. En SylvieDidou y Etienne Gérard (edit). Fuga de cerebros, movilidad académica y redes científicas, 117-136. México: IESALC-CINVESTAV-IRD. Recuperado de https://www.researchgate.net/publication/279751888_La_movilidad_academica_y_ estudiantil_reflexiones_sobre_el_caso_argentino.

González, J. y Salgado, C. (2016). Impacto de los programas de movilidad internacional en la adquisición de competencias académicas para el ingreso al mercado laboral: México. RIDE. Revista Iberoamericana para la Investigación y el Desarrollo Educativo, 7(13). Recuperado de http://www.scielo.org.mx/scielo.php?script=sci_arttext\&pid=S200774672016000200514.

González, A. (2011). Atrayendo talento: Estrategias de movilidad de los profesionales altamente cualificados en España. Sociología y Tecno ciencia 1(2), 72-87. Recuperado de https://dialnet.unirioja.es/servlet/articulo?codigo=3665173.

Sampieri, R., Fernández, C. y Baptista, P. (2010). Metodología de la investigación, México D.F.: McGraw-Hill.

Schwartzman, S. (2009). Nacionalismo versus Internacionalismo en las políticas de formación de recursos humanos de alto nivel. En SylvieDidou y Etienne Gérard (edit). Fuga de cerebros, movilidad académica y redes científicas, 63-74. México: IESALC-CINVESTAVIRD. 


\section{Original}

MOVILIDAD ESTUDIANTIL NACIONAL: INCLINACIÓN DE LOS UNIVERSITARIOS HACIA LA TOMA DE DECISIONES.

Tejada, G. (2012). Movilidad, conocimiento y cooperación: Las diásporas científicas como agentes del desarrollo. Centro de cooperación y desarrollo, 10(18), 1-24. Recuperado de http://www.scielo.org.mx/scielo.php?script=sci_arttext\&pid=S1870-75992012000100003.

Universidad Juárez Autónoma de Tabasco. (2015). Plan de desarrollo a largo plazo 2028. México: Colección Justo Sierra. Recuperado de http://www.archivos.ujat.mx/2016/PlanDesarrolloLargoPlazo2028/PLAN_2028_FINAL.pdf

Universidad Juárez Autónoma de Tabasco. (2018). Tercer Informe de Actividades. México: Colección Justo Sierra. Recuperado de http://www.ujat.mx/rectoria/24286. 